\title{
Optimal global carbon management with ocean sequestration
}

\author{
By Wilfried Rickels ${ }^{\star}$ and Thomas S. Lontzek†
}

${ }^{\star}$ Kiel Institute for the World Economy, Hindenburgufer 66, 24105, Kiel, Germany; e-mail: wilfried.rickels@ifw-kiel.de

†University of Zurich, Zurich, Switzerland

\begin{abstract}
We investigate the socially optimal intervention in the global carbon cycle. Limiting factors are (i) increasing atmospheric carbon concentration due to fossil fuel-related carbon emissions, and (ii) the inertia of the global carbon cycle itself. Accordingly, we explicitly include the largest non-atmospheric carbon reservoir, the ocean, to achieve a better representation of the global carbon cycle than the proportional-decay assumption usually resorted to in economic models. We also investigate the option to directly inject $\mathrm{CO}_{2}$ into the deep ocean (a form of carbon sequestration), deriving from this a critical level for ocean sequestration costs. Above this level, ocean sequestration is merely a temporary option; below it, ocean sequestration is the long-term option permitting extended use of fossil fuels. The latter alternative involves higher atmospheric stabilization levels. In this connection it should be noted that the efficiency of ocean sequestration depends on the time-preference and the inertia of the carbon cycle.
\end{abstract}

JEL classifications: Q30, Q54.

\section{Introduction}

Today, society has recognized the far-reaching consequences of the increase in atmospheric carbon concentration to above its preindustrial level because this contributes to a large extent to global warming. Nevertheless, the carbon concentration in the atmosphere is growing continuously and its growth rate increased even further in the period 2000-2006 (Canadell et al., 2007). This growth depends not only on global economic activity and the carbon intensity of the economy but also on the effectiveness of the natural carbon sinks, namely the terrestrial biosphere and the ocean. The uptake of the terrestrial biosphere is subject to strong fluctuations that are not well understood yet. Looking at the change in the various carbon reservoirs from 1800 to 1994, Sabine et al. (2004) show that the terrestrial 
biosphere has been a total net source of $39( \pm 28)$ Gt C. ${ }^{1}$ They conclude that 'the ocean has constituted the only true net sink for anthropogenic $\mathrm{CO}_{2}$ over the past 200 years' (p.370). The absolute value of the long-run atmospheric carbon stabilization level and the time pattern of its achievement will depend crucially on the marine carbon cycle, which is therefore perceived to be the most import cycle with regard to the climate (Najjar, 1992). Therefore, any approach to mitigate global warming that ignores the ocean ignores optimization potential.

In this paper, we address the question how the inclusion of the largest carbon reservoir of the carbon cycle, the ocean, changes the optimal path of carbon emissions and whether its inclusion allows additional optimization potential. Since natural forces transport carbon into the deep ocean, where it cannot affect society as adversely as when in the atmosphere, the logical question is: at which cost level would it be beneficial to accelerate the process of downward carbon transfer by injecting carbon into the deep ocean? $\mathrm{CO}_{2}$ could be transported via pipelines or ships to an ocean storage site, where it would be injected into the water column of the ocean or at the seafloor. This way, it would also become part of the global carbon cycle, but would enter the cycle in a more favorable way (Marchetti, 1977; Ozaki et al., 2001; IPCC, 2005; Keeling, 2009).

The growing knowledge about the importance of the marine carbon cycle for the mitigation of global warming has not just led to the inclusion of the oceanic carbon sink in the pioneering integrated assessment model (see, e.g., modifications from DICE 94 to DICE 99, Nordhaus, 1994; Nordhaus and Boyer, 2000, respectively). It has also led to the development of highly sophisticated computer models that include complex coupled atmosphere-ocean general circulation models to represent the global carbon cycle and the climate system of the world (for an overview of integrated assessment models, see, e.g., Kelly and Kolstad, 1999; Tol, 2006). However, many general properties of the stock externality problem were derived in microeconomic optimal control models, like Plourde (1976) or Forster (1980). In the application of these optimal control models to climate change problems, the global carbon cycle is only roughly approximated. The majority of the models apply a constant rate of decay, which yields a proportional decay of carbon in the atmosphere. As a result, in these models, global warming presents itself merely as a problem of temporary duration and the atmospheric carbon stock is represented as a completely renewable resource (see, e.g., Tahvonen, 1997). The other extremum is to model the atmospheric carbon stock without decay, whereby it becomes a completely non-renewable resource (see, e.g., Section 4 in Hoel, 1978; or Farzin, 1996). The atmospheric carbon stock is neither appropriately represented by a completely renewable description nor by a completely non-renewable description. Whereas the completely renewable description clearly overestimates the

\footnotetext{
${ }^{1}$ Based on the net increase of $165 \mathrm{Gt} \mathrm{C}$ in the atmosphere from 1800 to 1994, Sabine et al. (2004) subtract their ocean inventory estimate of $118( \pm 19)$ Gt $C$ from the total of fossil-fuel-emitted carbon, which amounted to $244( \pm 20)$ Gt $C$ during this period. As a consequence, the terrestrial biosphere has to be considered a net source of carbon if the carbon budget is to be balanced.
} 
storing capacity of the global carbon cycle, the completely non-renewable description underestimates the storing capacity of the global carbon cycle. The first description implies a complete oceanic carbon sink, the second description neglects the oceanic carbon sink. Taking into account this oceanic carbon uptake, the atmospheric carbon stock could be considered as a partial non-renewable resource. This approach is followed by Farzin and Tahvonen (1996). Based on a paper by Maier-Raimer and Hasselmann (1987), they divide the atmospheric carbon stock within their dynamic system artificially into two different stocks, one with a constant rate of decay and the other without. Given that the proportion of emissions to the non-decaying stock is equal to the long-run equilibrium, Farzin and Tahvonen's model captures important aspects of the carbon cycle. Nevertheless, the only management option in their dynamic model is to control the amount of emissions released into the atmosphere, which is proportional to the amount of extracted fossil fuels. Herzog et al. (2003) consider the injection of $\mathrm{CO}_{2}$ into the deep ocean. They calculate the effectiveness of this activity, measured as the ratio between the net benefit gained from temporary storage and the benefit gained from permanent storage. They find 'that the value of relatively deep ocean sequestration is nearly equivalent to permanent sequestration if marginal damage (i.e., carbon prices) remains constant or if there is a backstop technology that caps the abatement cost in the not too distant future' (p.306). However, their calculation of ocean sequestration is not embedded within an optimal control framework, and therefore is not the result of a combined extraction and sequestration decision.

We analyse the optimal amount of extraction and consumption of fossil fuels whereby the related emissions can be released into the atmosphere and injected into the deep ocean for purposes of ocean sequestration in a microeconomic partial analysis framework. In Section 2 we explain how we include the oceanic carbon stock in the optimization problem by applying a two-box model representation for the global carbon cycle. Thereby, we replace the constant or nonconstant decay assumption and capture the essential nonrenewable aspects of the global carbon cycle without artificially dividing the atmospheric carbon stock. In Section 3 we presents our results. In Section 3.1 we derive the general optimality conditions for the solution, before we start in Section 3.2 by analysing the scenario, where the fossil-fuel related emissions can only be released to the atmosphere. In Section 3.3 we analyse the scenario where fossil-fuel related carbon emission can also be injected into the deep ocean and derive a critical level for ocean sequestration costs. In Section 3.4 we analyse the policy relevant case, where the start-up costs of ocean sequestration are below the critical level and the initial levels of atmospheric carbon concentration are still below atmospheric stabilization targets. Finally, Section 4 concludes.

\section{Anthropogenic intervention into the global carbon cycle}

We investigate the optimal anthropogenic intervention into the carbon cycle in the light of global warming as a social planner's problem in which the planer needs to 
determine the global optimal amount of fossil fuels extraction and consumption with related emissions released to the atmosphere, $q(t)$, and the global optimal amount of fossil fuels extraction and consumption with related emissions injected into the deep ocean for purpose of sequestration, $a(t)$. Consequently, the total amount of fossil fuels extraction and consumption is $x(t)=q(t)+a(t)$. The social welfare function can be formalized as follows:

$$
\begin{gathered}
\max _{q(t), a(t)} \int_{0}^{\infty}(U(q(t)+a(t))-A(a(t))-D(S(t))) e^{-\rho t} d t, \\
\text { with } a(t), q(t) \geq 0
\end{gathered}
$$

which has to be maximized subject to the constraints:

$$
\begin{gathered}
\dot{S}=q(t)-\gamma(S(t)-\omega W(t)) \quad \text { with } \quad S\left(t_{0}\right)=S_{0}, \\
\dot{W}=a(t)+\gamma(S(t)-\omega W(t)) \quad \text { with } \quad W\left(t_{0}\right)=W_{0}, \\
\dot{R}=-q(t)-a(t) \quad \text { with } \quad R\left(t_{0}\right)=R_{0} .
\end{gathered}
$$

The total amount of fossil fuel extraction and consumption, $q(t)+a(t)$, generates gross utility in the social welfare function at any instant in time. The gross utility of total fossil fuel consumption is described by $U(x(t))$, which has the properties $U^{\prime}>0, U^{\prime \prime}<0$, and $U^{\prime}(0)=b<\infty$. The last property implies that there is a choke price or a backstop price. We assume that the costs of fossil fuel extraction are independent from the resource stock and are included in $U(x(t))$. The proportional amount of carbon emissions related to total fossil fuels consumption (the proportionality factor is one) can be released directly to the atmosphere, $q(t)$ (emissions), or injected into the deep ocean, $a(t)$ (ocean sequestration). Ocean sequestration generates additional costs in the social welfare function at any instant in time. The costs of ocean sequestration are described by $A(a(t))$, which has the properties $A^{\prime}>0$ and $A^{\prime \prime}>0$ and is measured in the same units as utility. Ocean sequestration summarizes the activities of capturing $\mathrm{CO}_{2}$ generated from the use of fossil fuels, of transporting the captured $\mathrm{CO}_{2}$ via pipelines or ships to an ocean storage site, and of injecting it into the deep ocean. The IPCC (2005) special report on carbon dioxide capture and storage provides cost ranges for $\mathrm{CO}_{2}$ capture. The ranges indicate that sequestration costs vary by differences in the design of $\mathrm{CO}_{2}$ capture systems and by differences in the operating and financing of the reference plant to which the capture technology is applied (p.27). Additionally, their estimates show that the costs are increasing as the transportation distance on land and on sea increases (p.31, Figure TS.6, and p.39, Table TS.8). We assume that carbon capture would be first applied to the most cost efficient plants and to plants located nearest to the shore. However, in order to increase the amount of ocean sequestration, the capture technology has to applied to less efficient plants and to plants located far away from the shore. Consequently, by including ocean sequestration into such a microeconomic partial analysis framework such as ours, the most appropriate representation of the costs becomes a convex function. 


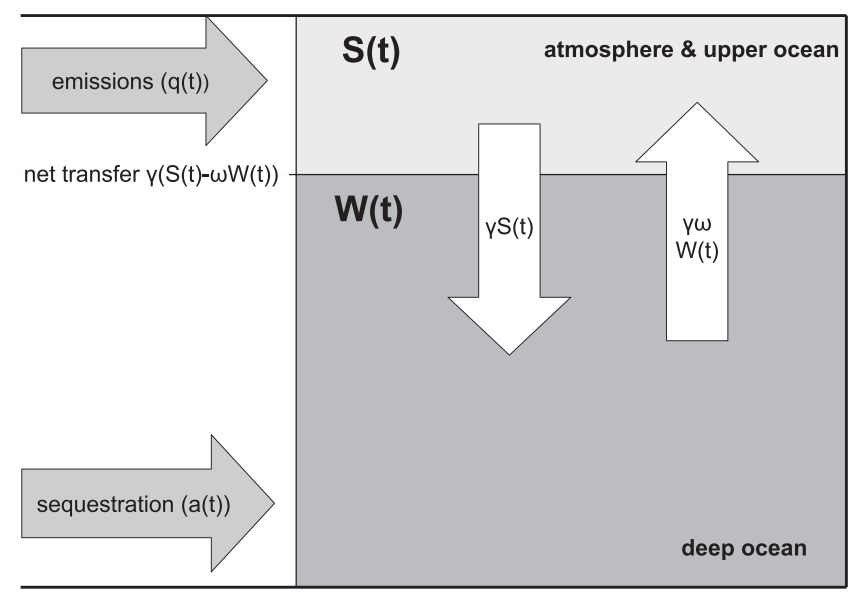

Fig. 1 Two-box model.

Both control variables, $q(t)$ and $a(t)$, increase the amount of carbon in the global carbon cycle, whereas only the atmospheric carbon stock influences the objective function. The increase in the atmospheric carbon stock to above pre-industrial levels leads to global warming and thereby causes social costs for society at any instant in time. The social costs of global warming are denoted as damage and are described by the strictly convex function $D(S(t))$, with the properties $D^{\prime}>0$, $D^{\prime \prime}>0$, and $D^{\prime}(0)=0$.

Equations (3) and (4) constitute the two-box model representation of atmosphere and ocean (see Fig. 1), whereby the boxes entail the carbon stocks in the atmosphere and the ocean, respectively. Equation (5) incorporates the endowment of the fossil resource, $R(t)$. Equations (3) to (5) describe the dynamics of the global carbon cycle as a consequence of the anthropogenic intervention. The upper box aggregates the carbon stocks in the atmosphere and in the upper mixed layer of the ocean. There is a net transfer of carbon between the atmosphere and the upper mixed layer of the ocean if there is a difference in the partial pressure of carbon dioxide $\left(\mathrm{pCO}_{2}\right)$ between these two reservoirs. The equilibration time for the upper layer of the ocean with the atmosphere takes around one year. ${ }^{2}$ However, only a small fraction of the ocean is involved in direct exchange with the atmosphere and the uptake bottleneck is the transport of anthropogenic carbon to the deeper parts of the ocean. Consequently, we assume that the atmosphere and the upper mixed

\footnotetext{
${ }^{2}$ Most of the $\mathrm{CO}_{2}$ dissolves in water, forming carbon acid first and then bicarbonate $\left(\mathrm{HCO}_{3}^{-}\right)$and carbonate ions $\left(\mathrm{CO}_{3}^{2-}\right)$. The sum of these three elements describes the total amount of carbon in the ocean, called dissolved inorganic carbon (DIC). The amount of DIC in the ocean consists to $89.1 \%$ of bicarbonate ions, to $10.4 \%$ of carbonate ions and only to $0.5 \%$ of $\mathrm{CO}_{2}$ (Najjar, 1992). Regarding the last figure, the atmosphere 'sees' only a tiny fraction of the carbon present in ocean surface water within the chemical process of $\mathrm{pCO}_{2}$ equilibration between the atmosphere and the ocean.
} 
layer are always in equilibrium and that the stock of carbon in the atmosphere is a constant fraction of the carbon stock in the upper box, $S(t)$.

In order to model the transport of anthropogenic carbon to the deeper parts of the ocean, we include the carbon stock in the deep ocean, $W(t)$, in the lower box. The transport of anthropogenic carbon to the deeper parts of the oceans is effected by the biological pump and especially by the solubility pump. The term biological pump designates the small fraction of organic matter and skeletons that survives remineralization in the euphotic zone and sinks to deeper layers. The main contribution to the transport of anthropogenic carbon is provided by the solubility pump. The solubility pump is driven by two phenomena: thermohaline circulation and the solubility of $\mathrm{CO}_{2}$. Surface water in equilibrium with atmospheric $\mathrm{CO}_{2}$ takes up additional $\mathrm{CO}_{2}$ on its way to the earth's poles, as the decreasing temperature increases the solubility of $\mathrm{CO}_{2}$. The formation of deep seawater is driven by thermohaline circulation, which transports cold and high-solubility high-latitude surface waters into the deep ocean. Consequently, these two phenomena act together to pump carbon from the atmosphere into the ocean's deeper layers until the deep ocean is saturated with respect to the upper layer. At this point in time, the up-welling water in the mid-latitudes transports anthropogenic carbon back to the upper layer.

The downward flux of carbon from the upper box to the lower box is represented by the fraction $\gamma S(t)$ and the upwards flux of carbon from the lower box to the upper box by the fraction $\gamma \omega W(t)$. These two fluxes are represented in Fig. 1 by the two white vertical arrows between the boxes. Both arrows have the same size, indicating that the upward flux is balanced by the downward flux. Putting these two fluxes together, we obtain the net transfer between the boxes, $\gamma(S(t)-\omega W(t))$. There will be a net flux between these two boxes if there is a difference between the relative stock sizes. An increase in the stock size in the upper box causes a downward transfer of excess carbon into the deep ocean, whereas up-welling water is still free of excess carbon, so that we observe a net transfer from the upper box into the lower box. The upper box is relatively small in comparison to the lower box. Consequently, $\omega$ is the proportionality factor to scale the stock of carbon in the lower box with respect to the upper box and $\gamma$ is the turnover factor to describe the speed of the adjustment process. ${ }^{3}$ The anthropogenic intervention into the carbon cycle, the amount of emissions and the amount of ocean sequestration are depicted by the grey horizontal arrows in Fig. 1. The amount of emissions enters the upper box and the amount of ocean sequestration enters the lower box. ${ }^{4}$ Even though the carbon stock in upper box entails atmospheric and oceanic carbon, we refer to it as the atmospheric carbon stock.

\footnotetext{
${ }^{3}$ Currently, this turnover speed is mainly limiting the uptake process by the ocean, so that the total ocean is estimated to be undersaturated for a long time (order of $10^{3}$ years) (Körtzinger and Wallace, 2002).

${ }^{4}$ Note it would also be possible to apply the control variables $x(t)$ and $a(t)$ instead of $q(t)$ and $a(t)$. As a result, only the net emissions, $x(t)-a(t)$, would be released to the upper box and the control constraints, (2), would change to $x(t)-a(t) \geqslant 0$ and $a(t) \geqslant 0$. Releasing the first constraint by
} 


\section{Results}

\subsection{Optimal solution conditions}

The corresponding current value Hamiltonian from (1) and (3) to (5) is

$$
\begin{aligned}
& H_{c}=U(q+a)-A(a)-D(S)-\psi \dot{S}-\pi \dot{W}+\mu \dot{R}, \\
& \text { where } \quad \lim _{t \rightarrow \infty} S(t) \geq 0, \quad \lim _{t \rightarrow \infty} W(t) \geq 0, \quad \lim _{t \rightarrow \infty} R(t) \geq 0 .
\end{aligned}
$$

Note that from now on we drop the time variable whenever it is convenient. We have changed the signs of the costate variables, $\psi$ and $\pi$, in order to facilitate their economic interpretation as taxes. Together with the two Lagrange multipliers for the control constraints (2), $\theta_{1}$ and $\theta_{2}$, we obtain the current value Lagrangian:

$$
L_{c}=H_{c}-\theta_{1}(-q)-\theta_{2}(-a) \text {. }
$$

According to Proposition 6.2 and Propostion 7.5 in Feichtinger and Hartl (1986), the admissible solution candidate has to fulfill the necessary conditions,

$$
\begin{aligned}
\frac{\partial L_{c}}{\partial q} & =0 \Rightarrow U^{\prime}-\psi-\mu+\theta_{1}=0, \\
\frac{\partial L_{c}}{\partial a} & =0 \Rightarrow U^{\prime}-A^{\prime}-\pi-\mu+\theta_{2}=0, \\
-\frac{\partial L_{c}}{\partial S} & =-\dot{\psi}+\rho \psi \Rightarrow-D^{\prime}(S)+\gamma \psi-\gamma \pi=\dot{\psi}-\rho \psi, \\
-\frac{\partial L_{c}}{\partial W} & =-\dot{\pi}+\rho \pi \Rightarrow-\gamma \omega \psi+\gamma \omega \pi=\dot{\pi}-\rho \pi, \\
-\frac{\partial L_{c}}{\partial R} & =\dot{\mu}-\rho \mu \Rightarrow 0=\dot{\mu}-\rho \mu, \\
\frac{\partial L_{c}}{\partial \theta_{1}} & \geq 0 \quad \theta_{1} \geq 0 \quad \theta_{1}(-q)=0, \\
\frac{\partial L_{c}}{\partial \theta_{2}} & \geq 0 \quad \theta_{2} \geq 0 \quad \theta_{2}(-a)=0,
\end{aligned}
$$

as well as the transversality conditions,

$$
\lim _{t \rightarrow \infty} e^{-\rho t} \psi=0, \quad \lim _{t \rightarrow \infty} e^{-\rho t} \pi=0, \quad \lim _{t \rightarrow \infty} e^{-\rho t} \mu R=0,
$$

and the constraint qualification for the control constraints (see Appendix 1). As any admissible path for the state and costate variables is non-negative and as any admissible path for the state variables is bounded due to the description of the carbon cycle as a closed system, the fulfillment of the transversality conditions, (16), is sufficient for the fulfillment of the general transversality conditions in a infinity 
horizon problem (Feichtinger and Hartl, 1986, Remark 2.9 and Remark 7.5). The fulfillment of the necessary conditions, (9) to (16), provides the optimal solution, because our carbon cycle is described by linear equations, (3) to (5), the control constraints (2) are quasi-concave, and in Appendix 1 we show that the maximized Hamiltonian is concave in the state variables and the Hamiltonian is strictly concave in the control variables (Feichtinger and Hartl, 1986, Proposition 7.5). The strict concavity of the Hamiltonian in the control variables implies that the Hamiltonian is regular and that therefore the controls are continuous, in particular at switching points (Feichtinger and Hartl, 1986, corollary 6.2). Note eqs (3) to (5) constitute a closed system, which means that no carbon vanishes from the cycle, $\dot{S}+\dot{W}+\dot{R}=0$ and one state variable, e.g., $W(t)=R_{0}+S_{0}+W_{0}-R(t)-S(t)$, and the corresponding costate variable can be dropped. Consequently, the modified Hamiltonian dynamic system with a full rank is a $4 \times 4$ dynamic system. The remaining two costate variables also measure then the influence of the omitted state variable on the objective function. To facilitate interpretation, the analysis is based on the system with all state variables (Full system), whereas some technical arguments and the calculations in the Appendix are based on the system with only two state variables (Redux system).

The optimal amounts of the control variables, $q$ and $a$, are determined by the costate variables, $\mu, \psi$, and $\pi$, where $\mu$ measures the shadow resource scarcity rent, $-\psi$ measures the shadow environmental scarcity rent of the atmospheric carbon stock and $-\pi$ measures the shadow environmental scarcity rent of the oceanic carbon stock (Farzin, 1996). The two costate variables $\psi$ and $\pi$ can be interpreted as the optimal tax values throughout time for an implementation of the social optimal solution in a decentralized economy. The costate variable that corresponds to the carbon stock in the upper box, $\psi$, denotes an emission tax, and the costate variable that corresponds to the carbon stock in the lower box, $\pi$, denotes an ocean sequestration tax. Conditions (11) and (12) indicate that both taxes are always positive and that the emission tax is always larger than the ocean sequestration tax, otherwise the transversality conditions (16) would be violated. This can be seen by solving the equation of motion for the tax difference, $\lambda=\psi-\pi$, which coincides with the emission tax in the Redux system, in which the state variable $W$ and the corresponding costate variable $\pi$ have been dropped, ${ }^{5} \psi_{R}$ :

$$
\dot{\lambda}=\lambda(\rho+\gamma+\gamma \omega)-D^{\prime}(S) \Rightarrow \lambda(t)=\psi_{R}(t)=\int_{t}^{\infty} D^{\prime}(S) e^{-(\rho+\gamma+\gamma \omega)(\tau-t)} d \tau .
$$

Consequently, if anthropogenic intervention takes place, the tax difference is positive. For an unconstrained solution, $\theta_{1}=\theta_{2}=0$, this can be seen directly by

\footnotetext{
${ }^{5}$ The costate variables $\mu_{R}$ and $\psi_{R}$ also measure the shadow environmental scarcity rent of the oceanic carbon stock. A lower $R$ implies ceteris paribus a higher oceanic carbon stock and therefore $\mu_{R}>\mu$ (sum of shadow resource scarcity rent and ocean sequestration tax). A lower $S$ implies ceteris paribus also a higher oceanic carbon stock but $\psi_{R}<\psi$ because the negative effect of a higher oceanic carbon stock is overcompensated by the positive effect of a lower atmospheric carbon stock (difference between emission and ocean sequestration tax). This can also be seen from (9) and (10) by dropping $\pi$ in (10).
} 
simplifying (10) to $A=\psi-\pi$. Both taxes, $\psi$ and $\pi$ are increasing in the atmospheric carbon stock as does the tax difference, indicating that the emission tax is increasing stronger than the ocean sequestration tax. ${ }^{6}$ The economic interpretation is that the amount of emissions directly increases the harmful carbon stock in the upper box, while the amount of ocean sequestration only indirectly does via the natural transfer. Nevertheless, as the ocean sequestration tax is positive, we see that ocean sequestration does cause social costs due to its temporary storage characteristics and therefore does not completely offset emissions into the atmosphere.

\subsection{Scenario 1: optimal extraction without the option of ocean sequestration}

We start investigating the implications of the representation of the global carbon cycle as a two-box model by considering a scenario where only one control variable is available, $q$, the extraction and consumption of fossil fuels with related emissions released to the atmosphere (Scenario 1). The anthropogenic intervention into the global carbon cycle ends when no further carbon is added due to the fact that either the marginal damage caused by carbon in the atmosphere has increased to such an extent that the choke price has been hit by the emission tax (Situation A) or the fossil resource stock is completely exploited (Situation B). In Situation A, the solution approaches a steady state as $t \rightarrow \infty$ and the steady state values for the costate variables, which fulfill the transversality conditions (16), $\operatorname{are}^{7}$

$$
\psi_{\infty}^{A 1}=\frac{D^{\prime}\left(S_{\infty}\right)}{\gamma+\rho+\gamma \omega}+\frac{\gamma \omega D^{\prime}\left(S_{\infty}\right)}{\rho(\gamma+\rho+\gamma \omega)}, \quad \pi_{\infty}^{A 1}=\frac{\gamma \omega D^{\prime}\left(S_{\infty}\right)}{\rho(\gamma+\rho+\gamma \omega)}, \quad \mu_{\infty}^{A 1}=0 .
$$

Using the steady state values for the costate variables, we can derive from (9), in which the Kuhn-Tucker multiplier, $\theta_{1}$, is zero, the level of the steady state atmospheric carbon stock:

$$
S_{\infty}^{A 1}=D^{\prime-1}\left(b \frac{\rho(\gamma+\rho+\gamma \omega)}{\rho+\gamma \omega}\right) .
$$

Equations (3) and (4) indicate that the two-box model is a non-renewable resource model, because no carbon vanishes or decays. Therefore, the release of carbon will increase the stock in both boxes forever and the two-box model approaches a new equilibrium with an atmospheric carbon stabilization level above the preindustrial one. The comparable atmospheric stabilization levels obtained with a renewable description of the global carbon cycle (R), like in Tahvonen (1997), obtained with a

\footnotetext{
${ }^{6}$ The increase in the emission tax follows directly from the interpretation of $-\psi$ being the shadow environmental scarcity rent of the atmospheric carbon stock, measuring the change in the value function due to a change in the corresponding state variable (see, e.g., Sydsæter et al., 2005). The increase in the tax difference follows from (17). The increase in the ocean sequestration tax follows from $\pi(t)=\gamma \omega \int_{t}^{\infty} \lambda e^{-\rho(\tau-t)} d \tau$.

${ }^{7}$ In the Redux system where the state variable $W$ and the corresponding state variable $\pi$ have been dropped, $\mu_{R}$ has a positive steady state value equal to the steady state value of $\pi_{\infty}^{A 1}$ in the Full system.
} 
non-renewable description of the global carbon cycle (NR), like in Hoel (1978), and obtained with a partially non-renewable description of the global carbon cycle (PNR), like in Farzin and Tahvonen (1996), are $^{8}$

$$
S_{\infty}^{R}=S_{0}, \quad S_{\infty}^{N R}=D^{\prime-1}(b \rho), \quad S_{\infty}^{P N R}=D^{\prime-1}\left(b \frac{\rho(\alpha+\rho)}{a-\alpha a+\rho}\right) .
$$

Intuitively, the renewable description implies that the atmospheric carbon stock returns to its preindustrial level when the release of carbon emissions has ended due to physical $\left(R_{\infty}=0\right)$ or economical $\left(R_{\infty} \geqslant 0\right)$ exhaustion of the fossil resource. The atmospheric stabilization levels of the non-renewable and partially non-renewable description are only compared if Situation A also applies, that is, if it is not optimal to completely exploit the fossil resource. The non-renewable description provides lower atmospheric carbon stabilization levels than the two-box model, because all carbon emissions remain in the atmosphere. The partially non-renewable description provides the same atmospheric stabilization levels as the two-box model if $a$ is chosen to be $\frac{\gamma+\omega}{\rho+\gamma+\omega}$ and $\alpha$ is chosen to be $\gamma$ in $S_{\infty}^{P N R}$, whereby the parameter $a$ describes the fraction of emissions that adds to the decaying carbon stock and the parameter $\alpha$ describes the fraction of the carbon stock that decays within the decaying carbon stock. The renewable description implies a complete oceanic carbon sink; the non-renewable description neglects the oceanic carbon sink. The reality is somewhere in between: 15\% (Körtzinger and Wallace, 2002) to 20\% (IPCC, 2005) of all anthropogenic $\mathrm{CO}_{2}$ will remain in the atmosphere within a new carbon cycle equilibrium. Consequently, the most appropriate description seems to be the partially non-renewable one. The comparison with the partially non-renewable description in Farzin and Tahvonen's model shows that the two-box model has no advantage in itself in representing the global carbon cycle. However, it becomes indispensable if further options to release carbon into the carbon cycle are considered (see Section 3.3).

The new equilibrium of the two-box model implies that there is no net transfer between the boxes and that $S_{\infty}=\omega W_{\infty}$ is fulfilled. Consequently, we can derive the critical initial level of the fossil resource for the steady state in Situation A as being feasible:

$$
R_{c r i t}^{A 1}=\left(\frac{\omega+1}{\omega}\right) D^{\prime-1}\left(b \frac{\rho(\gamma+\rho+\gamma \omega)}{\rho+\gamma \omega}\right)-S_{0}-W_{0} .
$$

If $R_{0}<R_{c r i t}^{A 1}$, extraction stops in finite time because $U^{\prime}(0)=b<\infty$ (Farzin and Tahvonen, 1996). The corresponding atmospheric and oceanic carbon stabilization levels are then

$$
S_{\infty}^{B 1}=\frac{\omega}{1+\omega}\left(R_{0}+S_{0}+W_{0}\right), \quad W_{\infty}^{B 1}=\frac{1}{1+\omega}\left(R_{0}+S_{0}+W_{0}\right),
$$

\footnotetext{
${ }^{8}$ In Farzin and Tahvonen's model, they divide the atmospheric carbon stock artificially into two different stocks, one with a constant rate of decay and the other without. Their objective function includes stock-dependent extraction costs as well, which we have set to zero in order to compare the steady states.
} 
which are lower than the carbon stabilization levels in Situation A. Note the stabilization levels in Situation B are not approached at the point in time when extraction stops, but as $t \rightarrow \infty$. According to Meinshausen et al. (2009), the emission of carbon from all proven fossil fuel resources would exceed the atmospheric stabilization levels corresponding to a $2^{\circ} \mathrm{C}$ temperature increase above preindustrial levels, which has been accepted by most countries as maximum tolerable limit for global warming. We focus therefore in our analysis on Situation A, where the limiting factor for optimal extraction is not the endowment of the fossil resource, but rather harmful levels of atmospheric carbon concentration, and impose therefore

Assumption $1 \quad R_{0} \geq R_{\text {crit }}^{A 1}$.

We show in Appendix 2 that the steady state of the $4 \times 4$ Hamiltonian dynamic system is a saddle point with four real eigenvalues, two being positive and two being negative. If Assumption 1 is fulfilled, the steady state is feasible and the optimal solution is the unique saddle path converging to the steady state as $t \rightarrow \infty$. Additionally, it can be seen from (13) and from the steady state levels of the costate variable in Situation A (18) that $\mu(t)=0$ for $t \epsilon[0, \infty)$. The fulfillment of Assumption 1 implies that the fossil resource is not scarce for the optimal solution in Situation A. The optimal path of extraction is therefore only determined by the emission tax, $\psi$.

Proposition 1 In Scenario 1 (related emissions can only be released to the atmosphere), if functional forms (9)-(11) are restricted to be quadratic-linear, Assumption 1 holds, and $U^{\prime}(0)=b<\infty$, the global optimal path for the emission tax, $\psi$, is either monotonically increasing or U-shaped.

Proof Because of the saddle path property with four real eigenvalues, two being positive and two being negative (see Appendix 2), the optimal path of the emission tax towards the steady state for quadratic-linear functional forms is determined by the two negative real eigenvalues in exponential terms and can therefore only entail one extremum. As a result, the set of possible paths is limited to a monotonically increasing, a monotonically decreasing, a U-shaped, and an inversely U-shaped path. The fulfillment of (9) in the steady state with $q_{\infty}=0$ requires $\psi_{\infty}=U^{\prime}(0)=$ $b \leqslant \infty$. Therefore, the control constraint $q(t) \geqslant 0$ for $t \epsilon[0, \infty)$ allows only tax paths that increase into the steady state.

Proposition 2 In Scenario 1 (related emissions can only be released to the atmosphere), if functional forms (9)-(11) are restricted to be quadratic-linear, Assumption 1 holds, and $U^{\prime}(0)=b<\infty$, the global optimal path for the ocean sequestration $\operatorname{tax}, \pi$, and the tax difference, $\lambda$, is either monotonically increasing or U-shaped.

Proof Again, the set of possible paths for the ocean sequestration tax and the tax difference is limited to a monotonically increasing, a monotonically decreasing, a $\mathrm{U}$-shaped, and an inversely $\mathrm{U}$-shaped path due to the saddle path property with real 
eigenvalues and the restriction to quadratic-linear functional forms. From (12), $\dot{\pi}=(\rho+\gamma \omega) \pi-\gamma \omega \psi$ it can be seen that it is not possible for $\pi$ to approach its steady state value from above. Such a path would imply that $\pi$ is decreasing, while the second term with $\psi$ is increasing due to Proposition 1. As a consequence, $\pi$ would continue to decrease. As both, $\psi$ and $\pi$ have to be increasing into the steady state so does $\lambda$ as it can be again seen from (12), $\dot{\pi}=\rho \pi-\gamma \omega \lambda$. Paths for $\pi$ and $\lambda$ which are decreasing do not allow to approach a steady state which in turn would violate the transversality condition (16).

Note, restricting functional forms to be quadratic-linear allows global statements for the tax paths in Proposition 1 and 2. Without the functional restriction, the propositions would only be locally in the neighborhood of the steady state valid. The two excluded paths from the set of possible paths, the monotonically decreasing and the inversely $\mathrm{U}$-shaped path, require an additional term that increases during the movement to the steady state so that (9) allows a declining amount of extraction even if the emission tax is decreasing. This requirement can be fulfilled by modeling extraction costs as not stock-independent, as in our model, but as stock-dependent, as in Farzin and Tahvonen (1996), $q C(R)$ with $C^{\prime}<0 .{ }^{9}$ The inclusion of stock-dependent extraction costs allows both physical exhaustibility $\left(R_{\infty}=0\right)$ and economical exhaustibility $\left(U^{\prime}(0)=C\left(R_{\infty}\right)\right.$ with $\left.R_{\infty} \geqslant 0\right)$ to be considered. However, our formulation with stock-independent extraction costs allows the implications of the description of the global carbon cycle to be clarified.

The renewable description of the global carbon cycle allows two tax paths, a monotonically decreasing and an inversely U-shaped path (e.g., Tahvonen, 1997). The non-renewable description of the global carbon cycle allows only one tax path, a monotonically increasing path (e.g., Farzin, 1996). The partially non-renewable description allows, compared to the non-renewable description, one additional possible path, a U-shaped path. Note Farzin and Tahvonen (1996) observe as well the two paths from the renewable description within their partially nonrenewable description, but not as consequence of the partially non-renewable description but due to the inclusion of stock-dependent extraction costs. The result is confirmed by the fact that the description with the two-box model allows the two additional paths from the renewable description to be observed as well if extraction costs are modeled to be stock-dependent (Lontzek and Rickels, 2008).

Additionally, Farzin and Tahvonen (1996) show that for the partially nonrenewable description and for a specific initial level of the atmospheric carbon stock, the possibility of a stationary emission tax with a stationary atmospheric carbon level to exist, given that there is no steady state, $U^{\prime}(0) \rightarrow \infty$. The stationary atmospheric carbon level requires that the decaying atmospheric carbon stock in the sum of the total atmospheric carbon stock declines at the rate at which the

\footnotetext{
${ }^{9}$ An alternative formulation is $C(X)$ with $C^{\prime}>0$, where $X$ measures the cumulative amount of extracted fossil fuels (Farzin, 1992).
} 
non-decaying atmospheric carbon stock increases. The decreasing decaying atmospheric carbon stock implies lower decay, so that extraction is decreasing at a constant rate. Such a stationary atmospheric carbon stock where the amount of emission is constantly decreasing is also possible with the two-box model. It requires that the amount of carbon emissions decreases at the same rate as the net transfer between the two boxes decreases due to the carbon accumulation in the deep ocean. The amount of emission would decline according to

$$
q(t)=\gamma \omega\left(\frac{\bar{S}}{\omega}-W_{0}\right) e^{-\gamma \omega t}
$$

and the oceanic carbon stock would increase according to

$$
W(t)=\frac{\bar{S}}{\omega}+\left(W_{0}-\frac{\bar{S}}{\omega}\right) e^{-\gamma \omega t},
$$

where $\bar{S}$ denotes the stationary atmospheric carbon level. Additionally, by changing our model formulation so that $U^{\prime}(0) \rightarrow \infty$ is valid and stock-dependent extraction costs are included, we could confirm that a constant emission tax occurs even with fossil fuel endowment as a specific feature of the description of the carbon cycle by a two-box model as it could be confirmed by Farzin and Tahvonen (1996) as a specific feature of the description of the carbon cycle by a partially non-renewable atmospheric carbon stock. However, the constant path requires, first, particular functional forms for $U(q)$ and $C(R)$ so that a constant percentage of the resource stock is extracted, which implies that extraction declines at this constant percentage rate (p.523), and, second, that the constant percentage coincides with the parameters of the carbon cycle description. ${ }^{10}$ Consequently, the partially nonrenewable description of the atmospheric carbon stock or the two-box model description of the carbon cycle is the precondition for such a path to occur, but the occurrence of such path is the consequence of a specific extraction path rather than the consequence of the carbon cycle description. In our analysis, we focus on the situation where the global carbon cycle approaches a steady state with non-constant taxes (Situation A).

\subsection{Scenario 2: optimal extraction with the option of ocean sequestration}

We turn to the scenario where both control variables, $q$ and $a$, are available, the extraction and consumption of fossil fuels with related emissions released to the atmosphere and the extraction and consumption of fossil fuels with related emissions injected into the deep ocean (Scenario 2). As in the previous section, the anthropogenic intervention into the global carbon cycle ends when no further carbon is added due to the fact that either the marginal damage caused by carbon in

\footnotetext{
${ }^{10}$ In Farzin and Tahvonen (1996) the constant percentage is given by $\alpha a$, the decay parameter and the fraction being added to the non-decaying stock, whereas in our model the constant percentage is given by the turnover speed between the boxes, $\gamma$, and the proportionality parameter, $\omega$.
} 
the atmosphere has increased to such an extent that the choke price has been hit or even exceeded by the emission tax (Situation A) or the fossil resource stock is completely exploited (Situation B). Again, in Situation A the solution approaches a steady state as $t \rightarrow \infty$ and the steady state values for the costate variables are given by (18) from Section 3.2. However, unlike in the previous section, not just (9) has to be fulfilled, but also (10) as well, which can be simplified to

$$
A^{\prime}+\theta_{1}-\theta_{2}=\psi-\pi \text {. }
$$

Condition (25) shows that the amount of ocean sequestration is determined by the difference between the two taxes, because by injecting carbon emissions into the ocean, one saves the emission tax, but instead has to pay the ocean sequestration tax. We already pointed out in the previous section that Situation A requires an increasing emission tax for $(9), U^{\prime}(0)=\psi_{\infty}$, to be fulfilled in the steady state. However, the amount of ocean sequestration is increasing in $\psi$ due to the convexity of the ocean sequestration cost function, and therefore we cannot further assume that the solution is unconstrained. Using the steady state levels of the taxes (18), we can derive two conditions ( $a$ and b) for the steady state atmospheric carbon stock to fulfill (9) and (10):

$$
\begin{aligned}
& S_{\infty}^{A 2 a}=D^{-1}\left(\left(b+\theta_{1}\right) \frac{\rho(\gamma+\rho+\gamma \omega)}{\rho+\gamma \omega}\right), \\
& S_{\infty}^{A 2 b}=D^{\prime-1}\left(\left(b-A^{\prime}(0)+\theta_{2}\right) \frac{\rho(\gamma+\rho+\gamma \omega)}{\gamma \omega}\right) .
\end{aligned}
$$

By equating $S_{\infty}^{A 2 a}$ and $S_{\infty}^{A 2 b}$, we obtain

$$
A^{\prime}(0)=b \frac{\rho}{\rho+\gamma \omega}-\frac{\gamma \omega}{\rho+\gamma \omega} \theta_{1}+\theta_{2},
$$

by which we distinguish three cases for the steady state: ${ }^{11}$

$$
\begin{array}{ll}
\text { Case 1: } & A^{\prime}(0)>b \frac{\rho}{\rho+\gamma \omega} \Rightarrow \theta_{1}=0 \quad \theta_{2}>0, \\
\text { Case 2: } & A^{\prime}(0)=b \frac{\rho}{\rho+\gamma \omega} \Rightarrow \theta_{1}=0 \quad \theta_{2}=0, \\
\text { Case 3: } & A^{\prime}(0)<b \frac{\rho}{\rho+\gamma \omega} \Rightarrow \theta_{1}>0 \quad \theta_{2}=0 .
\end{array}
$$

The value of $A^{\prime}(0)$ is somehow the counterpart of the choke price b. Whereas $b$ denotes the maximum value for marginal utility, $A^{\prime}(0)$ is the minimum level of the marginal sequestration costs. Therefore, $A^{\prime}(0)$ can be interpreted as the start-up

\footnotetext{
${ }^{11}$ We only consider carbon cycle equilibriums that are approached via anthropogenic intervention into the global carbon cycle and do not consider a potential Case 4 with both Kuhn-Tucker multipliers being positive, because in such a case the carbon cycle equilibrium is determined only by the initial levels, $S_{0}$ and $W_{0}$.
} 
cost for ocean sequestration. Due to the importance of $A^{\prime}(0)$ within Case 1 to 3 , we define

$$
A_{\text {crit }}=b \frac{\rho}{\rho+\gamma \omega}
$$

Proposition 3 In Scenario 2 (related emissions can be released to the atmosphere and injected into the deep ocean), if $A^{\prime}(0)<A_{\text {crit }}$ holds, the atmospheric stabilization level increases compared to Scenario 1.

Proof If $A^{\prime}(0) \geqslant A_{\text {crit }}, \theta_{1}$ is zero, (28) and (29), and we obtain

$$
S_{\infty}^{A 2 a}=S_{\infty}^{A 2 b}=S_{\infty}^{A 1}=D^{\prime-1}\left(b \frac{\rho(\gamma+\rho+\gamma \omega)}{\rho+\gamma \omega}\right) \quad \text { with } \quad \theta_{2}=A^{\prime}(0)-A_{\text {crit }} .
$$

If $A^{\prime}(0)<A_{\text {crit }}, \theta_{1}=\frac{b \rho-A^{\prime}(0)(\rho+\gamma \omega)}{\gamma \omega}>0, \theta_{2}$ is zero, and the function arguments in $S_{\infty}^{A 2 a}$ and $S_{\infty}^{A 2 b}$ increase uniformly and consequently the atmospheric stabilization level increases, so that $S_{\infty}^{A 2 a}=S_{\infty}^{A 2 b}>S_{\infty}^{A 1}$.

If the atmospheric carbon stabilization level increases compared to Scenario 1, so does the oceanic carbon stabilization level in order to satisfy the carbon cycle equilibrium condition $S_{\infty}=\omega W_{\infty}$. Consequently, we can again derive the critical initial level of the fossil resource for the steady state in Situation A as being feasible:

$$
R_{c r i t}^{A 2}\left(A^{\prime}(0)\right)=\left(\frac{\omega+1}{\omega}\right) D^{\prime-1}\left(\left(b-A^{\prime}(0)\right) \frac{\rho(\gamma+\rho+\gamma \omega)}{\gamma \omega}\right)-S_{0}-W_{0},
$$

whereas $R_{\text {crit }}^{2}\left(A^{\prime}(0)\right)>R_{\text {crit }}^{1}$ requires $A^{\prime}(0)<A_{\text {crit }}$ to be fulfilled. If $R_{0}<R_{\text {crit }}^{A 2}\left(A^{\prime}(0)\right)$, extraction stops again in finite time, as in Section 3.2, because $U^{\prime}(0)=b<\infty$ (Situation B). The corresponding atmospheric and oceanic carbon stabilization levels are only determined by the initial level of the fossil resource, $R_{0}$ (see (22)). It is possible to observe $R_{\text {crit }}^{A 2}\left(A^{\prime}(0)\right)>R_{0}>R_{\text {crit }}^{A 1}$ if $A^{\prime}(0)<A_{\text {crit }}$, so that the opportunity to inject carbon emissions related to fossil fuel consumption directly into the ocean would lead to the complete exploitation of the fossil resource (Situation B), whereas without this opportunity the fossil resource would not be completely exploited (Situation A). In Scenario 2, we also concentrate on Situation A and therefore extend Assumption 1,

Assumption $2 \quad R_{0} \geq R_{\text {crit }}^{A 2}\left(A^{\prime}(0)\right)$.

Again, if Assumption 2 is fulfilled, it can be seen from (13) and from the steady state level of the costate variable in Situation A (18) that $\mu(t)=0$ for $t \epsilon[0, \infty)$. The fulfillment of Assumption 2 implies that the fossil resource is not scarce for the optimal solution in Situation A. The optimal path of extraction is therefore determined only by the emission tax, $\psi$, and the ocean sequestration tax, $\pi$. The steady states in Case 1, (28), and Case 3, (30), have positive Kuhn-Tucker multipliers. Taking into account the continuity of the control variables, a point in time has to emerge, $t_{s}$, with $t_{s}<\infty$, after which the dynamic system is either described by 
$q(t) \geqslant 0$ and $a(t)=0$ for $t \epsilon\left[t_{s}, \infty\right)$ (Case 1$)$ or by $q(t)=0$ and $a(t) \geqslant 0$ for $t \epsilon\left[t_{s}, \infty\right)$ (Case 3 ) on the path towards the steady state. Note after $t_{s}$ the dynamic system leading toward the steady state coincides with a dynamic system which allows only extraction with related emissions released into the atmosphere (Case 1) or with a dynamic system which allows only extraction with related emissions injected into the deep ocean (Case 3). To formalize this idea, we define three control regimes: ${ }^{12}$

Regime 1: No sequestration: $a=0$ and $q \geqslant 0\left(\theta_{1}=0\right.$ and $\left.\theta_{2}>0\right)$,

Regime 2: Sequestration: $a \geqslant 0$ and $q \geqslant 0\left(\theta_{1}=0\right.$ and $\left.\theta_{2}=0\right)$,

Regime 3: Only sequestration: $a \geqslant 0$ and $q=0\left(\theta_{1}>0\right.$ and $\left.\theta_{2}=0\right)$.

Proposition 4 In Scenario 2 (related emissions can be released to the atmosphere and injected into the deep ocean), if Assumption 2 holds, and $U^{\prime}(0)=b<\infty$, the movement into the steady state is not an interior solution, but is, after some point in time, $t_{s}$, described either by Regime $1\left(A^{\prime}(0) \geqslant A_{\text {crit }}, R_{0}>R_{\text {crit }}^{A 2}\left(A^{\prime}(0)\right)=R_{\text {crit }}^{A 1}\right)$, or by Regime $3\left(A^{\prime}(0)<A_{\text {crit }}, R_{0}>R_{\text {crit }}^{A 2}\left(A^{\prime}(0)\right)>R_{\text {crit }}^{A 1}\right)$.

Proof Movement into the steady cannot be determined by Regime 2 because $q(t) \rightarrow 0$ requires an increasing emission tax, whereas $a(t) \rightarrow 0$ requires a decreasing emission tax so that the tax difference is also decreasing. Consequently, movement into the steady state implies at least one control constraint to be active (either $\theta_{1}$ or $\theta_{2}$ ).

We already referred in Section 3.2 to Appendix 2, where we show that Regime 1 (which is equal to Scenario 1), obeys saddle path properties with real eigenvalues. In Appendix 2 we also show that Regime 3 obeys saddle path properties. For the eigenvalues to be real in Regime 3, the condition

$$
\frac{1}{4}\left(\gamma(1+\omega)(\rho+\gamma+\gamma \omega)-D^{\prime \prime}\left(a_{\mu_{R}}^{\prime}\right)+a_{\pi_{R}}^{\prime}\right)^{2}>-\gamma \omega(\gamma+\rho+\gamma \omega) D^{\prime \prime} a_{\mu_{R}}^{\prime}
$$

has to be fulfilled in the steady state, where the subscript $R$ indicates the costate variables of the Redux system.

Proposition 5 In Scenario 2 (related emissions can be released to the atmosphere and injected into the deep ocean), if functional forms (9)-(11) are restricted to be quadratic-linear, Assumption 2 holds, and $U^{\prime}(0)=b<\infty$, the global optimal path for the emission and ocean sequestration tax after $t_{s}$ (final regime) is either monotonically increasing or U-shaped $\left(A^{\prime}(0) \geqslant A_{\text {crit }}\right)$ or monotonically increasing $\left(A^{\prime}(0)<A_{\text {crit }}\right)$.

Proof After point $t_{s}$, the optimal path is either determined by Regime 1 or Regime 3. We showed already in Propositions 1 and 2 that the optimal path is either monotonically increasing or U-shaped for Regime 1. For Regime 3, we see from (10), $U^{\prime}(a)-A^{\prime}(a)=\pi$, that only ocean sequestration tax paths that increase into

\footnotetext{
${ }^{12}$ We only consider control regimes with anthropogenic intervention into the global carbon cycle and do not consider a potential Regime 4 with $q=a=0\left(\theta_{1}>0\right.$ and $\left.\theta_{2}>0\right)$.
} 
the steady state fulfill the condition due to the concavity of the utility function and the convexity of the ocean sequestration cost function. We can therefore exclude paths that decrease into the steady state such as an inversely U-shaped path. Additionally, at $\left.t_{s}, \quad U^{\prime}\left(a\left(t_{s}\right)\right)=\psi\left(t_{s}\right), \quad U^{\prime}\left(a\left(t_{s}\right)\right)-A^{\prime}\left(a\left(t_{s}\right)\right)\right)=\pi\left(t_{s}\right)$, and $q\left(t_{s}\right)=0$ have to be fulfilled. We know from (12) that a U-shaped ocean sequestration tax requires a $U$-shaped emission tax for the transversality conditions to be fulfilled, (16). The decreasing emission tax $\psi(t)$ on the U-shaped path contradicts $q(t)=0$ for $t \epsilon\left[t_{s}, t^{\star}\right]$, where $t^{*}$ is defined by $\dot{\psi}\left(t^{*}\right)=0$ and $\ddot{\psi}\left(t^{*}\right)>0$, because $a(t)$ would be decreasing for $t \epsilon\left[t_{s}, t^{\star}\right]$ due to the decreasing tax difference (see Proposition 2) and therefore the LHS in $U^{\prime}(a(t))=\psi(t)$ would be increasing whereas the RHS would be deceasing. Consequently, if $A^{\prime}(0)<A_{\text {crit }}$ only monotonically increasing tax paths are possible for $t \epsilon\left[t_{s}, \infty\right]$.

Proposition 5 is only valid for $t \epsilon\left[t_{s}, \infty\right)$. Before the point $t_{s}$ is reached, various successions of regimes are possible, so that the possible set of optimal emission and ocean sequestration tax paths becomes more complex. Additionally, the dynamics in the regimes before the final regime are no longer determined by just the negative eigenvectors, but by the full set of eigenvectors. The reason for this is that the negative eigenvectors describe the optimal path towards the steady state corresponding to the regime (saddle path). However, in regimes prior to the final regime, the corresponding steady state is not feasible, and as a result the path towards such a non-feasible steady state cannot describe the optimal path towards the regime switching point.

\subsection{Utilizing ocean sequestration within a global carbon management strategy}

Consider the situation where the initial values for atmospheric and oceanic carbon stocks, $S_{0}$ and $W_{0}$, are low, the initial value for the fossil resource, $R_{0}$, fulfills Assumption 1, and the start-up costs for ocean sequestration are at least equal to the critical level, $A^{\prime}(0) \geqslant A_{\text {crit }}$. In this situation, the optimal solution is completely described by Regime 1 for $t \epsilon[0, \infty)$. The tax difference between the emission and ocean sequestration tax is never sufficient to bear the additional costs of ocean sequestration. The tax difference, which determines the amount of ocean sequestration, $A^{\prime}(a)=\psi-\pi$, does not reach the critical level, $A_{\text {crit }}$, before the steady state, so that with $A^{\prime}(0) \geqslant A_{\text {crit }}$ ocean sequestration is not beneficial. Obviously, if the initial levels for the carbon stocks are low, there is no difference between ocean sequestration that is too costly $\left(A^{\prime}(0) \geqslant A_{\text {crit }}\right.$ in Scenario 2$)$ and ocean sequestration that is not available or prohibited (Scenario 1). We refer to this situation as Policy 1.

Consider the situation where the initial values for atmospheric and oceanic carbon stocks, $S_{0}$ and $W_{0}$, are low, the initial value for the fossil resource stock, $R_{0}$, fulfills Assumption 2, and the start-up costs for ocean sequestration are below the critical level, $A^{\prime}(0)<A_{\text {crit }}$. In this situation, the optimal solution might be described by a succession of various regimes, but each succession involves 
Regime 3 for $t \epsilon\left[t_{s}, \infty\right)$. Even though a succession of various regimes is possible before $t_{s}$, the non-renewable description of the carbon cycle by the two-box model and the low initial levels imply tax paths that are increasing in direction towards $t_{s}$. Consequently, the tax difference will be at some point in time, $t<t_{s}$, above the start-up costs for ocean sequestration. Then it becomes beneficial to pay the ocean sequestration costs for some fraction of the emissions but to save the emission tax (Regime 1 to Regime 2). With the tax difference increasing into the steady state, the overall amount of fossil fuel consumption decreases, but the fraction of ocean sequestration for the related emissions increases. Consequently, at $t_{s}$ the complete amount of emissions related to fossil fuel consumption is injected into the deep ocean (Regime 2 to Regime 3 ). We see that from $t_{s}$ onwards, ocean sequestration is declining until $b-\pi_{\infty}=A^{\prime}(0)$ is fulfilled and that the emission tax increases above the choke price, $b=\psi_{s s}-\theta_{1}$ with $\theta_{1}>0$. The increasing tax paths prevent backward regime switches, e.g., from Regime 2 to Regime 1. Consequently, if ocean sequestration is not too costly, more than one regime can occur in the optimal solution. We refer to this situation as Policy 2.

In Fig. 2 we show the dynamics of the atmospheric and oceanic carbon stock, the emission and ocean sequestration tax and the controls for Policy 1 and Policy 2 for low initial levels by using simple quadratic-linear functional forms. ${ }^{13}$ Policy 2 shows the succession from Regime 2 to Regime 3. We see in the upper left graph that the atmospheric carbon stock increases slower in the beginning with Policy 2 than with Policy 1 . With Policy 2 carbon emissions are not only released into the atmosphere but are also injected into the deep ocean, consequently, the oceanic carbon stock increases faster with Policy 2 than with Policy 1, where it only increases due to the natural carbon transfer (upper right graph). Due to a slower increase in atmospheric carbon concentration, fossil fuel consumption declines slower with Policy 2 than with Policy 1 (lower right graph). The fraction of ocean sequestration for the related carbon emissions increases until it reaches $100 \%$ and only fossil fuels which allow related carbon emissions to be captures and injected into the deep ocean are consumed. This occurs, before upper and lower box have equilibrated, which can seen by a slight temporary decrease in the atmospheric carbon stock. However, in the long run, the atmospheric carbon concentration increases more with Policy 2 due to the extended use of the fossil resource and by a positive net transfer from the ocean to the atmosphere. Both taxes are increasing with Policy 2 in the long run above the levels which are obtained with Policy 1.

Even though we observe in Fig. 2 with Policy 2 a switch from Regime 2 to Regime 3, the dynamics are not characterized by a significant increase in

\footnotetext{
${ }^{13}$ The utility function is $U(q)=b q-u_{2} q^{2}$, the ocean sequestration cost function is $A(a)=a_{1} a+a_{2} a^{2}$, and the damage function is $D(S)=v_{1}\left(s S-A_{\text {preind }}\right)^{2}$. As a result of the linear-quadratic functional forms, the start-up costs, $A^{\prime}(0)$, simplify to the parameter value $a_{1}$. The parameter values are $b=5 / 10, \gamma=1 / 10$, $\omega=1 / 10, \rho=3 / 100, a_{1}=1 / 4 \quad a_{2}=1 / 10, u_{2}=1 / 20, v_{1}=0.1, s=3 / 10$, and $A_{\text {preind }}=6 / 10, S_{0}=2, W_{0}=20$. These parameter values yield $a_{\text {crit }}=3 / 8$ and $R_{\text {crit }}^{A 2}\left(a_{1}\right)=64.1667$.
} 

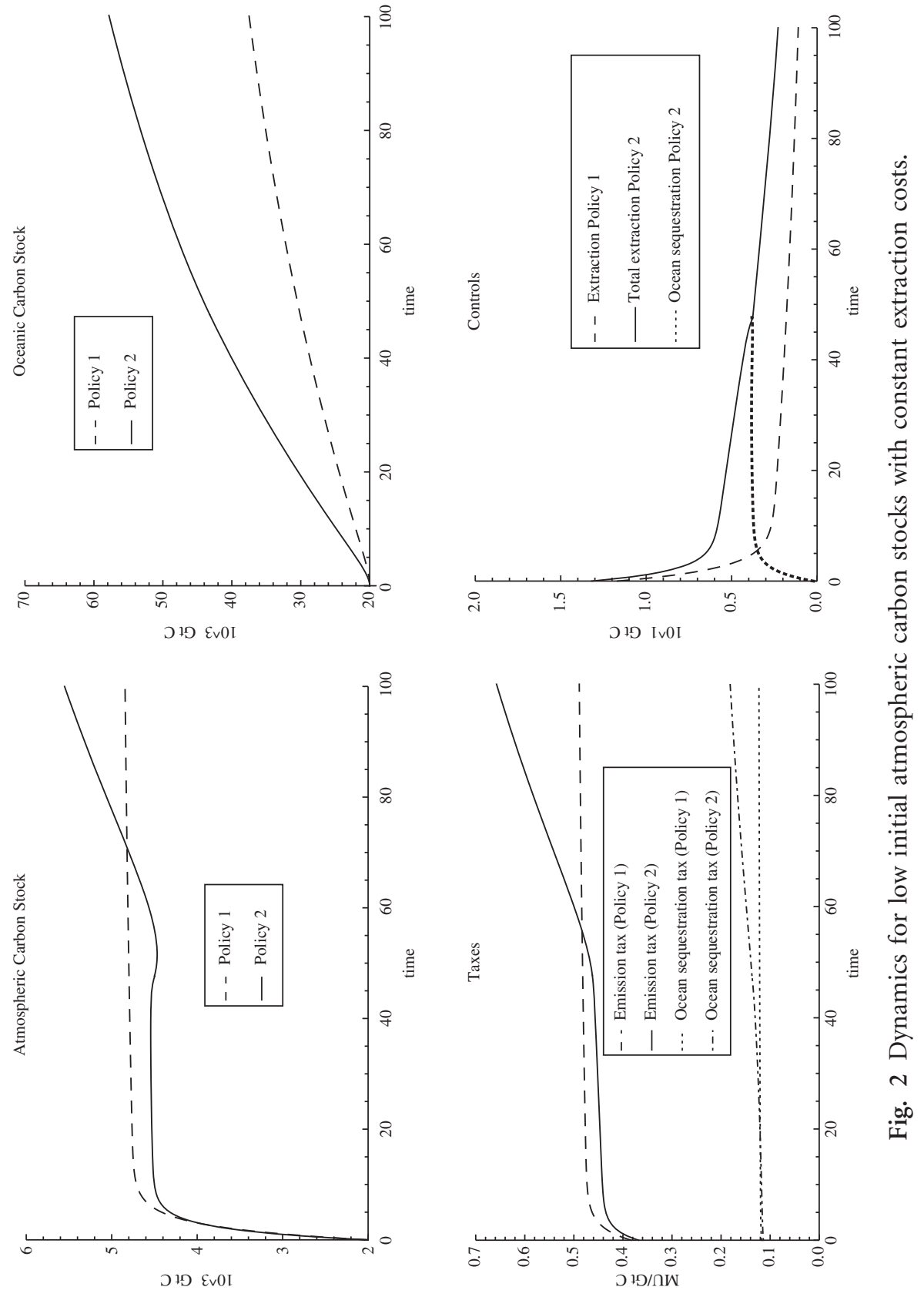
volatility. One reason for this is that the long-run dynamics are mainly influenced by the natural transfer parameters, $\gamma$ and $\omega$, which are rather low in order to represent the inertia of the carbon cycle. Consequently, the eigenvalues have a different magnitude and the Hamiltonian dynamic system is rather stiff. To demonstrate this effect, in Fig. 3 we again show Policy 1 and Policy 2 with a switch from Regime 2 to Regime 3, but this time with stock-dependent extraction costs included. ${ }^{14}$ However, instead of the emission tax and the ocean sequestration tax we show in Fig. 3 the difference between the two taxes. Due to the presence of stock-dependent extraction costs, the use of fossil fuels cannot be extended through ocean sequestration as it can in the case without stock-dependent extraction costs. Consequently, the atmospheric and oceanic stabilization levels are not affected by the magnitude as in Fig. 2. Total fossil fuel consumption in Scenario 2 is rather similar to fossil fuel consumption in Scenario 1 and at some point in time even slightly lower (lower right graph). Note that even with rather similar paths for total fossil fuel consumption, the atmospheric peak concentration is significantly lower due to the presence of ocean sequestration (upper left graph). Additionally, we see that both the atmospheric carbon stock and tax difference show a inverted S-shape with Policy 2 and confirm that the inclusion of ocean sequestration extends the set of possible tax paths so far discussed in the literature. The influence of ocean sequestration on atmospheric peak concentration and also the possibility of an interior solution due to inversely U-shaped tax paths are investigated in Lontzek and Rickels (2008).

The situation where the initial values for the atmospheric carbon, $S_{0}$, stock is high, whereas the initial level of the oceanic carbon stock, $W_{0}$, is low and the initial value for the fossil resource stock, $R_{0}$, fulfills Assumption 2 remains to be briefly considered. In this situation, the optimal solution might be described by a succession of various regimes, even with $A^{\prime}(0) \lesseqgtr A_{\text {crit }}$. In contrast to the non-renewable atmospheric carbon stock models (e.g. Hoel, 1978; Farzin, 1996), the non-renewable two-box model allows periods of time where the atmospheric carbon stock is decreasing. A decreasing atmospheric carbon stock implies that the natural downward transfer into the deep ocean exceeds the amount of emissions released into the atmosphere. If the harmful carbon stock in the atmosphere is initially high, the emission tax starts at a high initial level so that only small amounts of emissions are released into the atmosphere and the atmospheric carbon stock can equilibrate with the oceanic carbon stock due to the natural transfer while the emission tax is declining. However, whereas high emission tax levels imply low extraction with related emissions released to the atmosphere, they favor the utilization of ocean sequestration for the related emissions.

\footnotetext{
${ }^{14}$ The stock-dependent extraction cost function is $c_{1}-c_{2}{ }^{*} R(t)$, with the parameter values $c_{1}=5 / 10$, $c_{2}=1 / 200$, and $R_{0}=100$. Note the parameter value for $a_{1}$ has to be smaller than $1 / 8$ in order to still observe a final Regime 3, because, with the stock-dependent extraction costs, the critical level for the start-up costs changes to $A_{\text {crit }}=\left(b-C\left(R_{\infty}\right)\right) \frac{\rho}{\rho+\gamma \omega}$.
} 

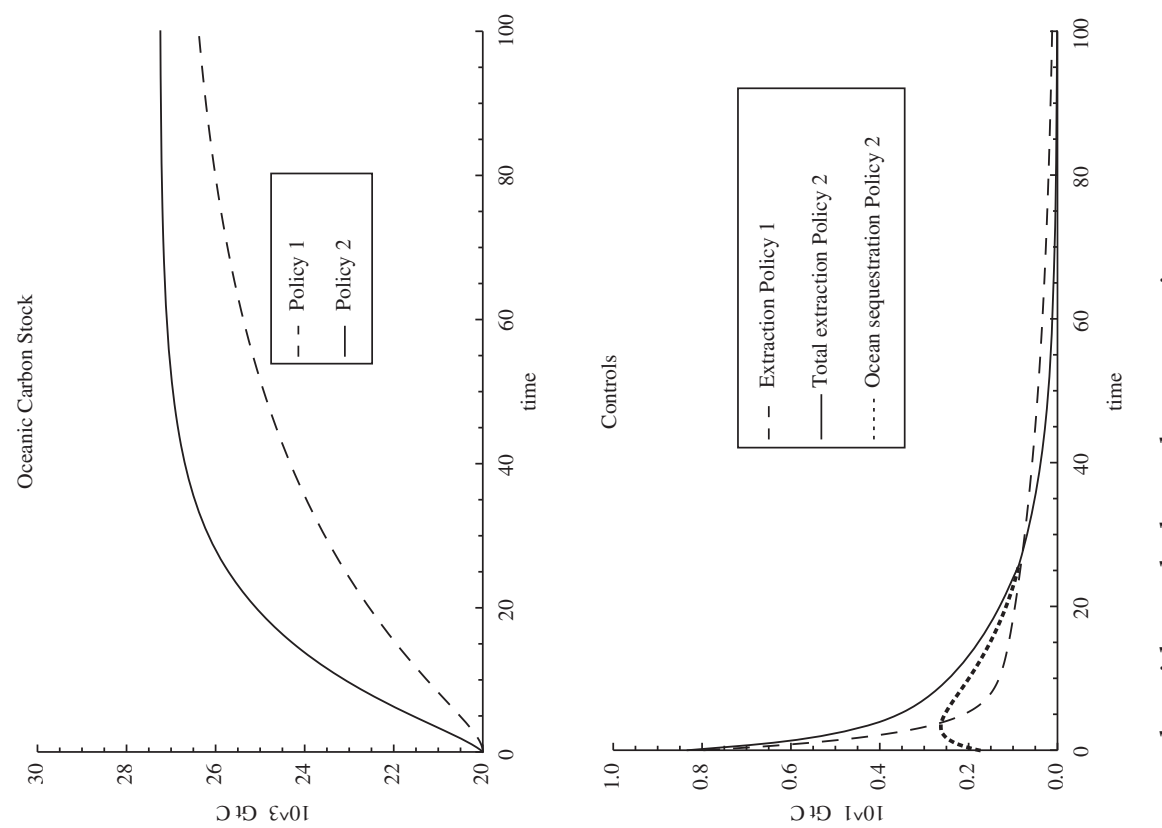

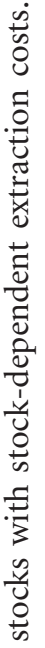
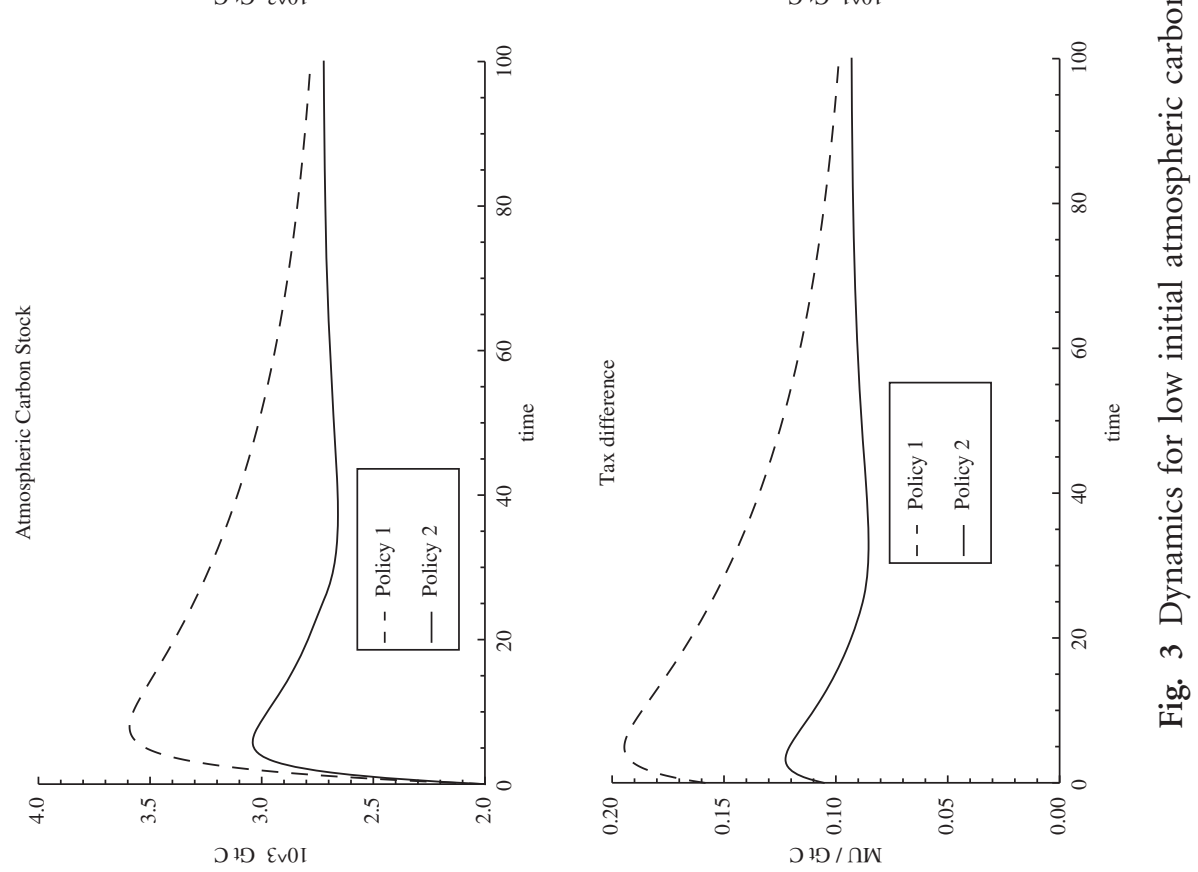
Consequently, given that the tax difference starts decreasing at a initial level that is higher than its steady state level, $\lambda(t)>\lambda_{\infty}$ for $t \epsilon\left[0, t_{a}\right)$, ocean sequestration is utilized if $\lambda(t) \geqslant A^{\prime}(0) \geqslant A_{\text {crit }}=\lambda_{\infty}$ for $t \epsilon\left[0, t_{a}\right)$ with $t_{a}$ defined by $\lambda\left(t_{a}\right)=\lambda_{\infty}$.

If the start-up costs are above the critical level, ocean sequestration can only be utilized until $t_{a}$, thereafter the dynamics are again complete described by Regime 1 . Consequently, if the initial levels for the atmospheric carbon stock are high, there is a difference between ocean sequestration that is too costly $\left(A^{\prime}(0)>A_{\text {crit }}\right.$ in Scenario 2) and ocean sequestration that is not available or prohibited (Scenario 1). Even though ocean sequestration is not an option for the long-run management of the global carbon cycle because it is too costly, it might be beneficial to utilize ocean sequestration for some period of time for the consumption of fossil fuels if the atmospheric carbon stock is rather high, but the oceanic carbon stock is still rather low.

If the start-up costs are below the critical level, ocean sequestration will be utilized beyond $t_{a}$, but not necessarily as the only control option. If the emission tax decreases sufficiently along the U-shaped path, it might be beneficial to switch back to Regime 2 and release some of the emissions again to the atmosphere, or even switch further back to Regime 1 and release all of the emissions again into the atmosphere. However, as the atmospheric carbon stock will start increasing again at some point in time so will the emission tax and therefore the dynamic system will return to Regime 3 at $t_{s}$ if $A^{\prime}(0)<A_{\text {crit }}$.

Irrespective of the tax paths that are realized, the effectiveness of ocean sequestration in this two-box model depends crucially on generating utility by using fossil fuels while delaying the damage resulting from increased levels of carbon in the atmosphere. As result, the effectiveness of sequestration depends on the time preferences and the adjustment times of the two boxes. The critical level, $A_{\text {crit }}$, is determined by the discount factor and the adjustment parameters of the two-box model. When the discount factor decreases, the critical level also decreases. As a result, the effectiveness of sequestration decreases because, with a lower discount factor, delaying damage pays off less. When $\gamma$ and $\omega$ decrease, the critical level increases. As a result, the effectiveness of sequestration increases because, with lower adjustment factors, the adjustment time of the two boxes decreases. A smaller value of $\gamma$ implies a slower mixing of the two boxes. A smaller value of $\omega$ implies a greater lower box, which in turn implies that the lower box can contain greater amounts of carbon. In the context of our two-box model, variations in the amount of carbon active in the lower box can be used to approximate various injection depths for ocean sequestration. A deeper injection depth goes along with a greater lower box and a slower adjustment process. Therefore, the effectiveness of ocean sequestration depends on the injection depth.

\section{Conclusions}

In this paper, we investigated optimal intervention into the global carbon cycle. To capture the complete accumulation of carbon in the global carbon 
cycle, we included, besides the atmospheric carbon stock, the oceanic carbon stock in a two-box model representation. Using a two-box model to describe the global carbon cycle does not in itself provide optimal atmospheric stabilization levels that are different from models with a partially non-renewable description of the atmospheric carbon stock. However, factoring in ocean sequestration may do so. Thus it is important to account for the amount of carbon that 'decays' by including the oceanic sink in the lower box. By doing so, we could show that ocean sequestration does not serve as a complete offset for a carbon emission tax, but has a price itself, an ocean sequestration tax. Furthermore, we clarified the implications of the nonrenewable description of the carbon cycle and therefore also for the partial non-renewable description of the atmospheric carbon stock for the optimal tax paths.

By deriving the critical level for the start-up costs of ocean sequestration we could determine the role of ocean sequestration in a global carbon management strategy. For ocean sequestration start-up costs above this level, ocean sequestration is merely a temporary option given initial atmospheric carbon concentration is high; below this level, ocean sequestration is the long-term option permitting extended use of fossil fuels. The latter alternative accompanies higher atmospheric and oceanic stabilization levels.

Given a climate policy that formulates an atmospheric carbon stabilization goal, ocean sequestration cannot increase the total amount of fossil fuels that can be consumed. Carbon injected into the deep ocean in excess of the atmosphere-ocean equilibrium amount corresponding to the atmospheric stabilization goal is expected to leak back to the atmosphere, because the ocean becomes supersaturated in relation to the atmosphere. However, the option of ocean sequestration does extend the period of time in that fossil fuels can be extracted in reasonable amounts, whereas without ocean sequestration the amounts of extraction would have to decline much earlier due to the inertia of the carbon cycle. Consequently, ocean sequestration constitutes a serious option with which to buy time to deal with the atmospheric carbon accumulation problem. The effectiveness of this option depends on the injection depth of the sequestered carbon and the time preference of society.

\section{Acknowledgements}

We would like to thank Andreas Oschlies, Paul Kramer, Toste Tanhua, Till Requate, Martin Quaas, two anonymous referees and associate editor Simon Cowan for helpful comments and suggestions. The usual caveats apply.

\section{Funding}

Cluster Proposal 2007/1 CP0718, Excellence Initiative 'Future Ocean', Kiel, by the Deutsche Forschungsgemeinschaft (DFG). 


\section{References}

Canadell, J., Quere, C.L., Raupach, M., Field, C., Buitehuis, E., Ciais, P., Conway, T., Gillett, N., Houghton, R., and Marland, G. (2007) Contributions to accelerating atmospheric $\mathrm{CO}_{2}$ growth from economic activity, carbon intensity, and efficiency of natural sinks, Proceedings of the National Academy of Sciences, 104, 18866-70.

Dockner, E. (1985) Local stability analysis in optimal control problems with two state variables, in G. Feichtinger (ed.) Optimal Control Theory and Economic Analysis 2, Elsevier Science Publishers, BV, Amsterdam.

Farzin, Y. (1992) The time path of scarcity rent in the theory of exhaustible resources, The Economic Journal, 102, 813-30.

Farzin, Y. (1996) Optimal pricing of environmental and natural resource use with stock externalities, Journal of Public Economics, 62, 31-57.

Farzin, Y. and Tahvonen, O. (1996) Global carbon cycle and the optimal time path of a carbon tax, Oxford Economic Papers, 48, 515-36.

Feichtinger, G. and Hartl, R.F. (1986) Optimale Kontrolle ökonomischer Prozesse, Walter de Gruyter, Berlin and New York.

Forster, B.A. (1980) Optimal energy use in a polluted environment, Journal of Environmental Economics and Management, 7, 321-33.

Herzog, H., Caldeira, K., and Reilly, J. (2003) An issue of permancence: assessing the effectiveness of temporary carbon storage, Climatic Change, 59, 293-310.

Hoel, M. (1978) Resource extraction and recycling with environmental costs, Journal of Environmental Economics and Management, 5, 220-35.

IPCC (2005) IPCC special report on carbon dioxide capture and storage, Cambridge University Press, Cambridge and New York, NY.

Keeling, R.F. (2009) Triage in the greenhouse, Nature Geosciences, 2, 820-22.

Kelly, D.L. and Kolstad, C.D. (1999) Integrated assessment models for climate change control, in H. Folmer and T. Tietenberg (eds) International Yearbook of Environmental and Resource Economics 1999/2000: A Survey of Current Issues, Ch.4. Edward Elgar, Cheltenham.

Körtzinger, A. and Wallace, D. (2002) Der globale Kohlenstoffkreislauf und seine anthropogene Störung - eine Betrachtung aus mariner Perspektive, promet, 28, 64-70.

Lontzek, T. and Rickels, W. (2008) Carbon capture and storage and the optimal path of the carbon tax, Working Paper 1475, Kiel Institute for the World Economy, Kiel.

Maier-Raimer, E. and Hasselmann, K. (1987) Transport and storage of $\mathrm{CO}_{2}$ in the ocean-an inorganic ocean-circulation carbon cycle model, Climate Dynamics, 2, 63-90.

Marchetti, C. (1977) On geoengineering and the $\mathrm{CO}_{2}$ problem, Climatic Change, 1, 59-68.

Meinshausen, M., Meinshausen, N., Hare, W., Raper, S.C., Frieler, K., Knutti, R., Frame, D.J., and Allen, M.R. (2009) Greenhouse-gas emission targets for limiting global warming to $2^{\circ} \mathrm{C}$, Nature, $458,1158-62$.

Najjar, R. (1992) Marine biogeochemistry, in K.E. Trenberth (ed.) Climate System Modeling, Cambridge University Press, Cambridge, 241-77.

Nordhaus, W.D. (1994) Managing the Global Commons: The Economics of Climate Change, MIT Press, Cambridge, MA, and London. 
Nordhaus, W.D. and Boyer, J. (2000) Warming the World: Economic Models of Global Warming, 1st edn, MIT Press, Cambridge, MA, and London.

Ozaki, M., Minamiura, J., Kitajima, Y., Mizokami, S., Takeuchi, K., and Hatakenaka, K. (2001) $\mathrm{CO}_{2}$ ocean sequestration by moving ships, Journal of Marine Science and Technology, 6, 51-8.

Plourde, C. (1976) A model of waste accumulation and disposal, The Canadian Journal of Economics, 5, 119-25.

Sabine, C., Feely, R., Gruber, N., Key, R., Lee, K., Bullister, J., Wanninkhof, R., Wong, C., Peng, T., Kozyr, A., Ono, T., and Rios, A. (2004) The oceanic sink for anthropogenic $\mathrm{CO}_{2}$, Science, 305, 367-71.

Sydsæter, K., Hammond, P., Seierstad, A., and Strøm, A. (2005) Further Mathematics for Economic Analysis, 1st edn, Prentice Hall, Pearson Education.

Tahvonen, O. (1989) On the Dynamics of Renewable Resource Harvesting and Optimal Pollution Control, The Helsinki School of Economics, Helsinki.

Tahvonen, O. (1997) Fossil fuels, stock externalities, and backstop technologies, The Canadian Journal of Economics, 30, 855-74.

Tol, R.S. (2006) Integrated assessment modelling, Working Papers FNU-102, Research unit Sustainability and Global Change, Hamburg University, Hamburg.

\section{Appendix 1: Necessary and sufficient optimality conditions}

For the two constraints, $g_{1}(q, a)=-q \leqslant 0$ and $g_{2}(q, a)=-a \leqslant 0$, the constraint qualification is fulfilled, if the matrix

$$
\left(\begin{array}{llll}
\frac{\partial g_{1}}{\partial q} & \frac{\partial g_{1}}{\partial a} & g_{1} & 0 \\
\frac{\partial g_{2}}{\partial q} & \frac{\partial g_{2}}{\partial a} & 0 & g_{2}
\end{array}\right)
$$

has the full row rank (Feichtinger and Hartl, 1986, p.161), which can be seen to be fulfilled from

$$
\left(\begin{array}{cccc}
-1 & 0 & -q & 0 \\
0 & -1 & 0 & -a
\end{array}\right)
$$

The concavity of the maximized Hamiltonian follows from the negative semidefiniteness of the Hessian matrix of the Hamiltonian (Feichtinger and Hartl, 1986, Remark 2.4). For the calculation of the Hessian matrix we eliminate the state variable $W(t)$ so that the carbon cycle eqs (3) to (5) simplify to

$$
\begin{gathered}
\dot{S}=q-\gamma\left(S-\omega\left(S_{0}+R_{0}+W_{0}-S-R\right)\right) \\
\dot{R}=-q-a .
\end{gathered}
$$

Consequently, the Current Value Hamiltonian is $H^{c}=U(q+a)-A(a)-D(S)-$ $\mu_{R} \dot{R}-\psi_{R} \dot{S}$, where the subscript $R$ indicates the costate variables of the Redux 
system, and we can calculate the Hessian matrix:

$$
\left(\begin{array}{cccc}
H_{S S} & H_{S R} & H_{S q} & H_{S a} \\
H_{R S} & H_{R R} & H_{R q} & H_{R a} \\
H_{q S} & H_{q R} & H_{q q} & H_{q a} \\
H_{a S} & H_{a R} & H_{a q} & H_{a a}
\end{array}\right)=\left(\begin{array}{cccc}
-D^{\prime \prime} & 0 & 0 & 0 \\
0 & 0 & 0 & 0 \\
0 & 0 & U^{\prime \prime} & U^{\prime \prime} \\
0 & 0 & U^{\prime \prime} & U^{\prime \prime}-A^{\prime \prime}
\end{array}\right),
$$

which has the eigenvalues

$$
\sigma_{1,2,34}=\left(\begin{array}{c}
-D^{\prime \prime} ; \frac{1}{2}\left(2 U^{\prime \prime}-A^{\prime \prime}-\sqrt{4\left(U^{\prime \prime}\right)^{2}+\left(A^{\prime \prime}\right)^{2}}\right), \\
\frac{1}{2}\left(-U^{\prime \prime}-A^{\prime \prime}+\sqrt{4\left(U^{\prime \prime}\right)^{2}+\left(A^{\prime \prime}\right)^{2}}\right), 0
\end{array}\right) .
$$

The Hessian matrix being negative semi-definite requires $\sigma_{1,2,3,4} \leqslant 0$. Taking into account our function properties, $A^{\prime \prime}>0, U^{\prime \prime}<0$, and $D^{\prime \prime}>0$, the negativeness of the third eigenvalue can be see from:

$$
A^{\prime \prime}-2 U^{\prime \prime}>\sqrt{\left(A^{\prime \prime}\right)^{2}+4\left(U^{\prime \prime}\right)^{2}}, \quad \text { and }-4 A^{\prime \prime} U^{\prime \prime}>0,
$$

whereas the first and second eigenvalue are obviously negative and the fourth eigenvalue is zero.

The regularity of the Hamiltonian follows from the strict concavity of the Hamiltonian in the control variables. The strict concavity can be seen from the lower right bloc matrix in the Hesse matrix (A5), because the first leading principal minor is negative $\left(U^{\prime \prime}<0\right)$ and the determinant of the lower right bloc matrix is positive, $-A^{\prime \prime} U^{\prime \prime}>0$.

\section{Appendix 2: Saddle path properties for Regime 1 and Regime 3}

Following Dockner (1985, Theorem 3) the fulfillment of first $K<0$ and second $0<\operatorname{Det}(M H D S)<(K / 2)^{2}$ is necessary and sufficient for the eigenvalues to be real, two being negative and two being positive. MHDS abbreviates modified Hamiltonian dynamic system and $K$ is defined as

$$
K=\operatorname{Det}\left(\begin{array}{cc}
\frac{\partial \dot{x}}{\partial x} & \frac{\partial \dot{x}}{\partial \lambda_{x}} \\
\frac{\partial \dot{\lambda}_{x}}{\partial x} & \frac{\partial \dot{\lambda}_{x}}{\partial \lambda_{x}}
\end{array}\right)+\operatorname{Det}\left(\begin{array}{cc}
\frac{\partial \dot{y}}{\partial y} & \frac{\partial \dot{y}}{\partial \lambda_{y}} \\
\frac{\partial \dot{\lambda}_{y}}{\partial y} & \frac{\partial \dot{\lambda}_{y}}{\partial \lambda_{y}}
\end{array}\right)+2 \operatorname{Det}\left(\begin{array}{cc}
\frac{\partial \dot{x}}{\partial y} & \frac{\partial \dot{x}}{\partial \lambda_{y}} \\
\frac{\partial \dot{\lambda}_{x}}{\partial y} & \frac{\partial \dot{\lambda}_{x}}{\partial \lambda_{y}}
\end{array}\right),
$$

where $x, y, \lambda_{x}$, and $\lambda_{y}$ denote state variables and the corresponding costate variables, respectively.

Regime 1 The MHDS for Scenario 1 as well as for Regime 1 is, again based on the system with full rank and eliminated state variable $W(t)$, where the subscript $R$ 
indicates the costate variables of the Redux system:

$$
\begin{aligned}
\dot{R} & =-q\left(\mu_{R}, \psi_{R}\right), \\
\dot{S} & =+q\left(\mu_{R}, \psi_{R}\right)-\gamma\left(S-\omega\left(S_{0}+R_{0}+W_{0}-S-R\right),\right. \\
\dot{\mu}_{R} & =\rho \mu_{R}-\gamma \omega \psi_{R}, \\
\dot{\psi}_{R} & =(\rho+\gamma+\gamma \omega) \psi_{R}-D^{\prime},
\end{aligned}
$$

and the corresponding Jacobian is

$$
J_{R 1}=\left(\begin{array}{cccc}
0 & 0 & -q_{\mu_{R}}^{\prime} & -q_{\psi_{R}}^{\prime} \\
-\gamma \omega & -\gamma-\gamma \omega & q_{\mu_{R}}^{\prime} & q_{\psi_{R}}^{\prime} \\
0 & 0 & \rho & -\gamma \omega \\
0 & -D^{\prime \prime} & 0 & \rho+\gamma+\gamma \omega
\end{array}\right) .
$$

We see that the determinate of $\operatorname{det}\left(J_{R 1}\right)=-\gamma^{2} \omega^{2} D^{\prime \prime} q_{\mu_{R}}^{\prime}-\gamma \rho \omega D^{\prime \prime} q_{\psi_{R}}$ and $K_{R 1}=$ $-\gamma(1+\omega)(\rho+\gamma+\gamma \omega)+D^{\prime \prime} q_{\psi_{R}}^{\prime}$ fulfill the conditions $K_{R 1}<0$ and $\operatorname{det}\left(J_{R 1}\right)>0$, because $q_{\psi_{R}}^{\prime}<0$ and $q_{\mu_{R}}^{\prime}<0$. Additionally, $\operatorname{det}\left(J_{R 1}\right)<(K / 2)^{2}$ is fulfilled, because

$$
(K / 2)^{2}-\operatorname{det}\left(J_{R 1}\right)=\gamma \omega D^{\prime \prime}\left(\gamma \omega q_{\mu_{R}}^{\prime}+\rho q_{\psi_{R}}^{\prime}+\frac{1}{4}(\gamma(1+\omega)(\rho+\gamma+\gamma \omega))-D^{\prime \prime} q_{\psi_{R}}^{\prime}\right)^{2}>0 .
$$

Regime 3 The MHDS for Regime 3 is, again based on the system with full rank and eliminated state variable $S(t)$, where the subscript $R$ indicates the costate variables of the Redux system:

$$
\begin{aligned}
\dot{R} & =-a\left(\mu_{R}, \pi_{R}\right), \\
\dot{W} & =a\left(\psi_{R}, \pi_{R}\right)+\gamma\left(S_{0}+R_{0}+W_{0}-R-W\right)-\gamma \omega W, \\
\dot{\mu}_{R} & =\rho \mu_{R}-\gamma \pi_{R}-D^{\prime}, \\
\dot{\pi}_{R} & =(\rho+\gamma \omega) \pi_{R}-D^{\prime},
\end{aligned}
$$

and the corresponding Jacobian is

$$
J_{R 3}=\left(\begin{array}{cccc}
0 & 0 & -a_{\mu_{R}}^{\prime} & -a_{\pi_{R}}^{\prime} \\
-\gamma & -\gamma \omega & a_{\mu_{R}}^{\prime} & a_{\pi_{R}}^{\prime} \\
D^{\prime \prime} & D^{\prime \prime} & 0 & -\gamma \\
D^{\prime \prime} & D^{\prime \prime} & 0 & \rho+\gamma \omega
\end{array}\right)
$$

We see that the determinate of $\operatorname{det}\left(J_{R 3}\right)=-\gamma \omega(\rho+\gamma+\gamma \omega) D^{\prime \prime} a_{\mu_{R}}^{\prime}$ and $K_{R 3}=$ $-\gamma(1+\omega)(\rho+\gamma+\gamma \omega)+D^{\prime \prime}\left(a_{\mu_{R}}^{\prime}+a_{\pi_{R}}^{\prime}\right)$ fulfill the conditions $\operatorname{det}\left(J_{R 3}\right)>0$ and $K_{R 3}<0$, because $a_{\mu_{R}}^{\prime}<0$ and $a_{\pi_{R}}^{\prime}<0$. Additionally, $\operatorname{det}\left(J_{R 1}\right)<(K / 2)^{2}$ is fulfilled, if

$$
\frac{1}{4}\left(\gamma(1+\omega)(\rho+\gamma+\gamma \omega)-D^{\prime \prime}\left(a_{\mu_{R}}^{\prime}\right)+a_{\pi_{R}}^{\prime}\right)^{2}>-\gamma \omega(\gamma+\rho+\gamma \omega) D^{\prime \prime} a_{\mu_{R}}^{\prime}
$$

is fulfilled in the steady state. If (A14) is not fulfilled, the saddle path property is not affected, but the eigenvalues are complex (Tahvonen, 1989). 